
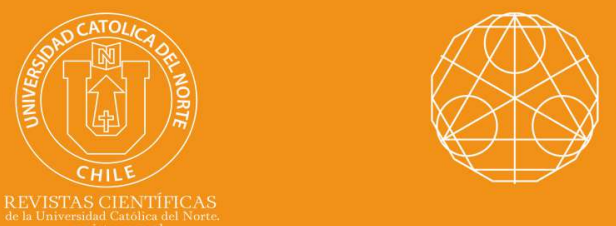

\title{
New algebraic properties of middle Bol loops II
}

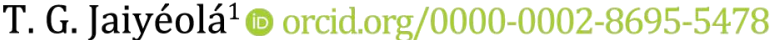

S. P. David ${ }^{2}$

O. O. Oyebola ${ }^{3}$ (1) orcid.org/0000-0001-8514-7367

Obafemi Awolowo University, Dept. of Mathematics, Ilé-Ifẹ, Nigeria.

1@ tjayeola@oauife.edu.ng; $2 \llbracket$ davidsp4ril@yahoo.com

${ }^{3}$ Federal University of Agriculture, Dept. of Mathematics, Abeokuta, Nigeria.

voyebolaoo@funaab.edu.ng

\section{Abstract:}

A loop $(Q, \cdot, 1, /)$ is called a middle Bol loop $(M B L)$ if it obeys the identity $x(y z \mid x)=(x / z)(y \mid x)$. To every $M B L$ corresponds a right $B o l$ loop (RBL) and a left Bol loop ( $L B L)$. In this paper, some new algebraic properties of a middle Bol loop are established in a different style. Some new methods of constructing a MBL by using a nonabelian group, the holomorph of a right Bol loop and a ring are described. Some equivalent necessary and sufficient conditions for a right (left) Bol loop to be a middle Bol loop are established. A RBL ( $M B L, L B L, M B L)$ is shown to be a $M B L(R B L, M B L, L B L)$ if and only if it is a Moufang loop.

Keywords: Bol loops; Middle Bol loops; Moufang loops.

MSC (2020): 20N02, 20N05.

\section{Cite this article as (IEEE citation style):}

T. G. Jaiyéolá, S. P. David, and O. O. Oyebola, "New algebraic properties of middle Bol loops II", Proyecciones (Antofagasta, On line), vol. 40, no. 1, pp. 85-106, 2021, doi: 10.22199/issn.0717-6279-2021-01-0006

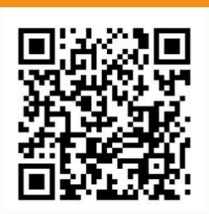

Article copyright: (C) 2021 T. G. Jaiyéolá, S. P. David, and 0. O. Oyebola. This is an open access article distributed under the terms of the Creative Commons License, which permits unrestricted use and distribution provided the original author and source are credited. 


\section{Introduction}

Let $G$ be a non-empty set. Define a binary operation $(\cdot)$ on $G$. If $x \cdot y \in G$ for all $x, y \in G$, then the pair $(G, \cdot)$ is called a groupoid or Magma. If each of the equations:

$$
a \cdot x=b \quad \text { and } \quad y \cdot a=b \quad \text { for all } a, b \in G
$$

has unique solutions in $G$ for $x$ and $y$ respectively, then $(G, \cdot)$ is called a quasigroup.

If there exists a unique element $e \in G$ called the identity element such that for all $x \in G, x \cdot e=e \cdot x=x$, then $(G, \cdot)$ is called a loop. We write $x y$ instead of $x \cdot y$, and stipulate that $\cdot$ has lower priority than juxtaposition among factors to be multiplied. For instance, $x \cdot y z$ stands for $x(y z)$.

Let $x$ be a fixed element in a groupoid $(G, \cdot)$. The left and right translation maps of $G, L_{x}$ and $R_{x}$ respectively can be defined by

$$
y L_{x}=x \cdot y \quad \text { and } \quad y R_{x}=y \cdot x .
$$

It can now be seen that a groupoid $(G, \cdot)$ is a quasigroup if its left and right translation mappings are bijections or permutations. Since the left and right translation mappings of a loop are bijective, then the inverse mappings $L_{x}^{-1}$ and $R_{x}^{-1}$ exist. Let

$$
x \backslash y=y L_{x}^{-1}=y \mathcal{L}_{x}=x \mathcal{R}_{y} \quad \text { and } \quad x / y=x R_{y}^{-1}=x \mathbf{R}_{y}=y \mathbf{L}_{x}
$$

and note that

$$
x \backslash y=z \Longleftrightarrow x \cdot z=y \quad \text { and } \quad x / y=z \Longleftrightarrow z \cdot y=x .
$$

Hence, $(G, \backslash)$ and $(G, /)$ are also quasigroups. Using the operations $(\backslash)$ and $(/)$, the definition of a loop can be stated as follows.

Definition 1.1. (i) $x \cdot(x \backslash y)=y,(y / x) \cdot x=y$ for all $x, y \in G$,

(ii) $x \backslash(x \cdot y)=y,(y \cdot x) / x=y$ for all $x, y \in G$ and

(iii) $x \backslash x=y / y$ or $e \cdot x=x$ for all $x, y \in G$.

We also stipulate that $(/)$ and $(\backslash)$ have higher priority than $(\cdot)$ among factors to be multiplied. For instance, $x \cdot y / z$ and $x \cdot y \backslash z$ stand for $x(y / z)$ and $x(y \backslash z)$ respectively. 
In a loop $(G, \cdot)$ with identity element $e$, the left inverse element of $x \in G$ is the element $x J_{\lambda}=x^{\lambda} \in G$ such that

$$
x^{\lambda} \cdot x=e
$$

while the right inverse element of $x \in G$ is the element $x J_{\rho}=x^{\rho} \in G$ such that

$$
x \cdot x^{\rho}=e .
$$

For an overview of the theory of loops, readers may check $[2,3,4$, $6,10,14,16,22]$.

A loop satisfying the identical relation

$$
(x y \cdot z) y=x(y z \cdot y)
$$

is called a right $\mathrm{Bol}$ loop (Bol loop, RBL). A loop satisfying the identical relation

$$
(x \cdot y x) z=x(y \cdot x z)
$$

is called a left Bol loop (LBL). A loop satisfying the identical relation

$$
x y \cdot z x=x(y z) \cdot x
$$

is called a Moufang loop.

A loop $(Q, \cdot)$ is called a middle Bol if it satisfies the identity

$$
x(y z \backslash x)=(x / z)(y \backslash x)
$$

It is known that the identity (1.4) is universal under loop isotopy and that the universality of (1.4) implies the power associativity of the middle Bol loops (Grecu and Syrbu [7]. Furthermore, (1.4) is a necessary and suffi- cient condition for the universality of the anti-automorphic inverse property (Syrbu [20]). Middle Bol loops were originally introduced in 1967 by Be- lousov [1] and were later considered in 1971 by Gwaramija [9], who proved that a loop $(Q, \circ)$ is middle Bol if and only if there exists a right $\operatorname{Bol} \operatorname{loop}(Q, \cdot)$ such that

$$
x \circ y=\left(y \cdot x y^{-1}\right) y, \text { for every } x, y \in Q .
$$

This result of Gwaramija [9] is formally stated below: 
Theorem 1.1. If $(Q, \cdot)$ is a left (right) Bol loop then the groupoid $(Q, \circ)$, where $x \circ y=y\left(y^{-1} x \cdot y\right)$ (respectively, $x \circ y=\left(y \cdot x y^{-1}\right) y$ ), for all $x, y \in Q$ , is a middle Bol loop and, conversely, if $(Q, \circ)$ is a middle Bol loop then there exists a left (right) Bol loop $(Q, \cdot)$ such that $x \circ y=y\left(y^{-1} x \cdot y\right)$ (respectively, $x \circ y=\left(y \cdot x y^{-1}\right) y$ ), for all $x, y \in Q$.

Remark 1.1. Theorem 1.1 implies that if $(Q, \cdot)$ is a left Bol loop and $(Q, \circ)$ is the corresponding middle Bol loop then $x \circ y=x / y^{-1}$ and $x \cdot y=x / / y^{-1}$ , where "/" ("//") is the right division in $(Q, \cdot)$ (respectively, in $(Q, \circ)$ ). Similarly, if $(Q, \cdot)$ is a right Bol loop and $(Q, \circ)$ is the corresponding middle Bol loop then $x \circ y=y^{-1} \backslash y$ and $x \cdot y=y / / x^{-1}$, where " $"(" / / ")$ is the left (right) division in $(Q, \cdot)$ (respectively, in $(Q, \circ)$ ). Hence, middle Bol loops are isostrophs of left and right Bol loops.

If $(Q, \circ)$ is a middle Bol loop and $(Q, \cdot)$ is the corresponding left Bol loop, then $(Q, *)$, where $x * y=y \cdot x$, for every $x, y \in Q$, is the corresponding right Bol loop for $(Q, \circ)$. So, $(Q, \cdot)$ is a left Bol loop, $(Q, *)$ is a right $B o l$ loop and

$$
x \circ y=y\left(y^{-1} x \cdot y\right)=\left[y *\left(x * y^{-1}\right)\right] * y,
$$

for every $x, y \in Q$.

After then, middle Bol loops resurfaced in literature not until 1994 and 1996 when Syrbu $[18,19]$ considered them in-relation to the universality of the elasticity law.

In 2003, Kuznetsov ([13]), while studying gyrogroups (a special class of Bol loops) established some algebraic properties of middle Bol loop and designed a method of constructing a middle Bol loop from a gyrogroup. According to him, in a middle Bol loop $(Q, \cdot)$ with identity element $e$, the following are true.

1. The left inverse element $x^{\lambda}$ and the right inverse element $x^{\rho}$ to an element $x \in Q$ coincide: $x^{\lambda}=x^{\rho}$.

2. If $(Q, \cdot, e)$ is a left Bol loop and "/" is the right inverse operation to the operation ". ", then the operation $x \circ y=x / y^{-1}$ is a middle Bol loop $(Q, \circ, e)$, and every one middle Bol loop can be obtained in a similar way from some left Bol loop. 
These confirm the observations of earlier authors mentioned above.

In 2010, Syrbu [20] studied the connections between structure and prop- erties of middle Bol loops and of the corresponding left Bol loops. It was noted that two middle Bol loops are isomorphic if and only if the corre- sponding left (right) Bol loops are isomorphic, and a general form of the autotopisms of middle Bol loops was deduced. Relations between different sets of elements, such as nucleus, left (right,middle) nuclei, the set of Mo- ufang elements, the center, e.t.c. of a middle Bol loop and left Bol loops were established.

In 2012, Grecu and Syrbu [7] proved that two middle Bol loops are isotopic if and only if the corresponding right (left) Bol loops are isotopic. They also proved that a middle Bol loop $(Q, \circ)$ is flexible if and only if the corresponding right $\mathrm{Bol}$ loop $(Q, \cdot)$ satisfies the identity

$$
(y x)^{-1} \cdot\left(x^{-1} \cdot y^{-1}\right)^{-1} x=x .
$$

In 2012, Drapal and Shcherbacov [5] rediscovered the middle Bol identities in a new way.

In 2013, Syrbu and Grecu [21] established a necessary and sufficient con- dition for the quotient loops of a middle Bol loop and of its corresponding right Bol loop to be isomorphic.

In 2014, Grecu and Syrbu [8] established:

1. that the commutant (centrum) of a middle Bol loop is an AIP-subloop and

2. a necessary and sufficient condition for the commutant to be invariant under the existing isostrophy between middle Bol loop and the corresponding right Bol loop.

In 1994, Syrbu[18], while studying loops with universal elasticity ( $x y$. $x=x \cdot y x)$ established a necessary and sufficient condition $(x y / z)(b \backslash x z)=$ $x(b \backslash[(b y / z)(b \backslash x z)])$ for a loop $(Q, \cdot, \backslash, /)$ to be universally elastic. Furthermore, he constructed some finite examples of loops in which this condition and the middle Bol identity $x(y z \backslash x)=(x / z)(y \backslash x)$ are equivalent, and then posed an open problem of investigating if these two identities are also equivalent in all other finite loops.

In 2012, Drapal and Shcherbacov [5] reported that Kinyon constructed a non-flexible middle Bol loop of order 16. This necessitates a reformulation of the Syrbu's open problem. Although the above authors also reported 
that Kinyon reformulated the Syrbu's open problem as follows: Let $Q$ be a loop such that every isotope of $Q$ is flexible and has the AAIP. Must $Q$ be a middle Bol loop? Jaiyéolá and David [11] initiated the preparation of the ground for different reformulation of Syrbu's open problem based on the fact that the algebraic properties and structural properties of middle Bol loops have been studied in the past relative to their corresponding right (left) Bol loop. In this work, we continue our earlier study

1. by digging out some new algebraic properties of a middle Bol loop;

2. developing new methods of constructing a middle Bol loop;

3. by establishing necessary and sufficient condition(s) for a right (left) Bol loop to be a middle Bol loop.

Jaiyéolá et al. [12] studied the holomorphy of middle Bol loops.

Definition 1.2. Let $(L, \cdot)$ be a loop. The the pair $(H, \circ)$ given by $H=$ $A(L) \times L$ where $A(L) \leq A U M(L, \cdot)$ and $(\alpha, x) \circ(\beta, y)=(\alpha \beta, x \beta \cdot y)$ for all $(\alpha, x),(\beta, y) \in H$ is a loop called the $A(L)$-Holomorph of $(L, \cdot)$.

We shall need the following results.

Lemma 1.1. (Jaiyéọlá and David [11]) Let $(Q, \cdot, \backslash, /)$ be a loop. The fol- lowing are equivalent.

1. $(Q, \cdot, \backslash, /)$ is a middle Bol loop.

2. $x(y z \backslash x)=(x / z)(y \backslash x) \quad$ for all $\quad x, y, z \in Q$.

3. $(x / y z) x=(x / z)(y \backslash x) \quad$ for all $\quad x, y, z \in Q$.

Theorem 1.2. (Pflugfelder [14])

A RBL $(L B L)(Q, \cdot)$ is a Moufang loop if and only if any of the following is true:

1. $(Q, \cdot)$ is a $L I P L(R I P L)$.

2. $(Q, \cdot)$ is a $L A P L(R A P L)$.

3. $(Q, \cdot)$ is a flexible loop.

4. $(Q, \cdot)$ is a $L B L(R B L)$. 
5. $(Q, \cdot)$ is a dissociative loop.

6. $(Q, \cdot)$ is a $A A I P L$.

Theorem 1.3. (Solarin and Sharma [17]) Let $H$ be a subgroup of a non- abelian group $G$ and let $A=H \times G$. For $\left(h_{1}, g_{1}\right),\left(h_{2}, g_{2}\right) \in A$,

define;

$$
\left(h_{1}, g_{1}\right) \circ\left(h_{2}, g_{2}\right)=\left(h_{1} h_{2}, h_{2} g_{1} h_{2}^{-1} g_{2}\right),
$$

then $(A, \circ)$ is a right Bol loop.

Theorem 1.4. (Robinson [15])

Let $(L, \cdot)$ be a loop with an $A(L)$-Holomorph $(H, \circ) .(H, \circ)$ is a right Bol loop if and only if

1. $(L, \cdot)$ is a right Bol loop and

2. $x^{-1} \cdot x \alpha \in N_{\rho}(L, \cdot) \quad$ for all $\quad \alpha \in A(L)$ and $x \in L$.

Consider $(G, \cdot)$ and $(H, \circ)$ being two groupoids(quasigroups, loops). Let $A, B$ and $C$ be three bijective mappings from $G$ onto $H$. The triple $\alpha=$ $(A, B, C)$ is called an isotopism of $(G, \cdot)$ onto $(H, \circ)$ if and only if

$$
x A \circ y B=(x \cdot y) C \forall x, y \in G .
$$

So, $(H, \circ)$ is called a groupoid(quasigroup, loop) isotope of $(G, \cdot)$.

If $C=I$, the identity map on $G$ so that $H=G$, then the triple $\alpha=(A, B, I)$ is called a principal isotopism of $(G, \cdot)$ onto $(G, \circ)$ and $(G, \circ)$ is called a principal isotope of $(G, \cdot)$. Eventually, the equation of relationship now becomes

$$
x \cdot y=x A \circ y B \quad \forall x, y \in G
$$

which is easier to work with. But taking $A=R_{g}$ and $B=L_{f}$ for some $f, g \in G$, the relationship now becomes

$$
x \cdot y=x R_{g} \circ y L_{f} \quad \forall x, y \in G \text { or } x \circ y=x R_{g}^{-1} \cdot y L_{f}^{-1} \quad \forall x, y \in G .
$$

With this new form, the triple $\alpha=\left(R_{g}, L_{f}, I\right)$ is called an $f, g$-principal isotopism of $(G, \cdot)$ onto $(G, \circ), f$ and $g$ are called translation elements of $G$ or at times written in the pair form $(g, f)$, while $(G, \circ)$ is called an $f, g$-principal isotope of $(G, \cdot)$.

The last form of $\alpha$ above gave rise to an important result in the study of loop isotopes of loops. 
Theorem 1.5. (Pflugfelder [14]) Let $(G, \cdot)$ and $(H, \circ)$ be two isotopic loops. For some $f, g \in G$, there exists an $f, g$-principal isotope $(G, *)$ of $(G, \cdot)$ such that $(H$, $\circ) \cong(G, *)$.

With this result, to investigate the isotopic invariance of an isomorphic invariant property in loops, one simply needs only to check if the property in consideration is true in all $f, g$-principal isotopes of the loop. A property is isotopic invariant if whenever it holds in the domain loop i.e $(G, \cdot)$ then it must hold in the co-domain loop i.e $(H, \circ)$ which is an isotope of the former. In such a situation, the property in consideration is said to be a universal property hence the loop is called a universal loop relative to the property in consideration. Like we mentioned earlier on, middle Bol loops are universal. by

Recall that for any quasigroup $(Q, \cdot)$, the group of autotopisms is given

$A U T(Q, \cdot)=\left\{T=(U, V, W) \in S Y M(Q)^{3} \mid x U \cdot y V=(x \cdot y) W \quad \forall x, y \in Q\right\}$.

We now introduce the set of anti-autotopisms giving by

$\operatorname{AAUT}(Q, \cdot)=\left\{T^{\prime}=<U^{\prime}, V^{\prime}, W^{\prime}>\in S Y M(Q)^{3} \mid x U^{\prime} \cdot y V^{\prime}=(y \cdot x) W^{\prime} \forall x, y \in Q\right\}$.

Define $\circ$ and $\odot$ on $S Y M(Q, \cdot)^{3}$ such that for all $\left[A_{1}, B_{1}, C_{1}\right],\left[A_{2}, B_{2}, C_{2}\right] \in$ $S Y M(Q)^{3}$ :

$$
\begin{gathered}
{\left[A_{1}, B_{1}, C_{1}\right] \circ\left[A_{2}, B_{2}, C_{2}\right]=\left[A_{1} A_{2}, B_{1} B_{2}, C_{1} C_{2}\right] \in S Y M(Q)^{3} \text { and }} \\
{\left[A_{1}, B_{1}, C_{1}\right] \odot\left[A_{2}, B_{2}, C_{2}\right]=\left[B_{1} A_{2}, A_{1} B_{2}, C_{1} C_{2}\right] \in S Y M(Q)^{3} .}
\end{gathered}
$$

\section{Main Results}

\subsection{Algebraic Properties of a Middle Bol Loop}

Theorem 2.1. Let $(Q, \cdot, \backslash, /)$ be a middle Bol loop. Then, for all $x, y, z, g \in$ $Q$ :
1. $x \cdot([y z] / g) \backslash(x g)=[(x g) / z][y \backslash(x g)]$.
2. $\left\langle\mathbf{L}_{x g}, \mathcal{R}_{x g}, \mathbf{R}_{g} \mathcal{R}_{x g} L_{x}\right\rangle \in A A U T(Q, \cdot)$.
3. $\mathbf{R}_{g} \mathcal{R}_{x g} L_{x}=\mathcal{R}_{x g} L_{x g}=\mathbf{L}_{x g} R_{x g}$.
4. $x \cdot(y / g) \backslash(x g)=(x g)[y \backslash(x g)]$. 
5. $x \cdot y \backslash(x g)=(x g)[(y g) \backslash(x g)]=(x g) /(y g) \cdot x g$.

6. $(y / x)^{-1}=x^{-1} \backslash y^{-1}$.

7. $(y / g) \backslash g=g(y \backslash g)$.

8. $x \cdot g^{-1} \backslash(x g)=(x g)^{2}$.

9. $g^{-1} \cdot x \backslash(x g)=x g$.

10. $(x y) \backslash(x \cdot x y)=y \backslash(x y)$.

Proof. Let $(Q, \cdot, \backslash, /)$ be a middle Bol loop with an arbitrary loop isotope $(H, \circ,$,$) . Then by Theorem 1.5, for some f, g \in G$, there exists $f, g$-principal isotope $(Q, *, \nwarrow, \nearrow)$ of $(Q, \cdot, \backslash, /)$ such that $(H, \circ,,) \cong(Q, *, \nwarrow, \nearrow)$. So,

$$
x \cdot y=x R_{g} \circ y L_{f} \Longleftrightarrow x * y=x R_{g}^{-1} \cdot y L_{f}^{-1}=x / g \cdot f \backslash y \quad \forall x, y \in G .
$$

$(Q, *, \nwarrow, \nearrow)$ is a middle Bol loop if and only if

$$
x *[(y * z) \nwarrow x]=(x \nearrow g) *(y \nwarrow x)
$$

It is easy to check that

$$
x \nearrow y=x /(f \backslash y) \cdot g \text { and } x \nwarrow y=f \cdot(x / g) \backslash y
$$

Applying (2.2) to the LHS and RHS of (2.1), we have:

$\mathrm{LHS}=\mathrm{x} *[(y * z) \nwarrow x]=x *[(y / g \cdot f \backslash z) \nwarrow x]=x *\{f \cdot[(y / g \cdot f \backslash z) / g] \backslash x\}$ $=x / g \cdot[(y / g \cdot f \backslash z) / g] \backslash x$.

$\mathrm{RHS}=(\mathrm{x} \nearrow g) *(y \nwarrow x)=[x /(f \backslash z) \cdot g] *[f \cdot(y / g) \backslash x]=[x /(f \backslash z)]$. $[(y / g) \backslash x]$.

Thus, LHS=RHS if and only if

$$
\begin{gathered}
x \cdot[(y z) / g] \backslash(x g)=[(x g) / z][y \backslash(x g)] \\
\Longleftrightarrow(y z) \mathbf{R}_{g} \mathcal{R}_{x g} L_{x}=z \mathbf{L}_{x g} \cdot y \mathcal{R}_{x g} \\
\Longleftrightarrow\left\langle\mathbf{L}_{x g}, \mathcal{R}_{x g}, \mathbf{R}_{g} \mathcal{R}_{x g} L_{x}\right\rangle \in \operatorname{AAUT}(Q, \cdot)
\end{gathered}
$$

Put $y=e, z=e$ in (2.4) separately to get 


$$
\begin{gathered}
\mathbf{R}_{g} \mathcal{R}_{x g} L_{x}=\mathcal{R}_{x g} L_{x g}=\mathbf{L}_{x g} R_{x g} \\
\Longleftrightarrow x \cdot y \backslash(x g)=(x g)[(y g) \backslash(x g)]=(x g) /(y g) \cdot x g
\end{gathered}
$$

So, we have proved 1. to 5 . according to (2.3) to (2.7) while the proof of 6 . to 10 . are achieved by making appropriate substitutions in (2.7).

Theorem 2.2. Let $(Q, \cdot, \backslash, /)$ be a middle Bol loop. Then, for all $x, y, z, f \in$ $Q$ :
1. $\{(f x) /[f \backslash(y z)]\} x=[(f x) / z][y \backslash(f x)]$.
2. $\left\langle\mathbf{L}_{f x}, \mathcal{R}_{f x}, \mathcal{L}_{f} \mathbf{L}_{f x} R_{x}\right\rangle \in A A U T(Q, \cdot)$.
3. $\mathcal{L}_{f} \mathbf{L}_{f x} R_{x}=\mathbf{L}_{f x} R_{f x}=\mathcal{R}_{f x} L_{f x}$.
4. $(f x) /(f \backslash y) \cdot x=(f x) / y=f x \cdot y \backslash(f x)$.
5. $(f x) / y \cdot x=(f x) /(f y) \cdot f x=f x \cdot(f y) \backslash(f x)$.
6. $(x \backslash y)^{-1}=y^{-1} / x^{-1}$.
7. $f /(f \backslash y)=f / y \cdot f,(f x) / f \cdot f x=f x \cdot x$.
8. $(f x)^{2}=(f x) / f^{-1} \cdot x$.
9. $(y x) / y=(y x \cdot x) /(y x)$.

Proof. The proof is similar to that of Theorem 2.1 based on the fact that by Lemma 1.1, identities (1.4) and $(x / y z) x=(x / z)(y \backslash x)$ equivalently define a MBL.

Lemma 2.1. Let $(Q, \cdot)$ be a quasigroup.
1. For all $T_{1}^{\prime}, T_{2}^{\prime} \in \operatorname{AAUT}(Q, \cdot), T_{1}^{\prime} \odot T_{2}^{\prime} \in \operatorname{AUT}(Q, \cdot)$.
2. $T^{\prime}=\langle U, V, W\rangle \in \operatorname{AAUT}(Q, \cdot) \Leftrightarrow T^{\prime-1}=\left\langle V^{-1}, U^{-1}, W^{-1}\right\rangle \in \operatorname{AAUT}(Q, \cdot)$.
3. For all $T \in A U T(Q, \cdot)$ and $T^{\prime} \in \operatorname{AAUT}(Q, \cdot), T \odot T^{\prime} \in \operatorname{AAUT}(Q, \cdot)$.
4. For all $T \in \operatorname{AUT}(Q, \cdot)$ and $T^{\prime} \in \operatorname{AAUT}(Q, \cdot), T^{\prime} \circ T \in A A U T(Q, \cdot)$.
5. For any anti-autotopism of $(Q, \cdot)$, any two components uniquely de- termine the third. 
Proof. Recall that

$A U T(Q, \cdot)=\{T=(U, V, W) \in S Y M(Q) \mid x U \cdot y V=(x \cdot y) W \quad \forall x, y \in Q\}$

and

$A A U T(Q, \cdot)=\left\{T^{\prime}=\left\langle U^{\prime}, V^{\prime}, W^{\prime}\right\rangle S Y M(Q) \mid x U^{\prime} \cdot y V^{\prime}=(y \cdot x) W^{\prime} \forall x, y \in Q\right\}$.

Then,

1. Let $T_{1}^{\prime}=\left\langle U^{\prime}, V^{\prime}, W^{\prime}\right\rangle, T_{2}^{\prime}=\left\langle U_{2}^{\prime}, V_{2}^{\prime}, W_{2}^{\prime}\right\rangle \in A A U T(Q, \cdot)$, then $x\left(V_{1}^{\prime} U_{2}^{\prime}\right) \cdot y\left(U_{1}^{\prime} V_{2}^{\prime}\right)=\left(x V_{1}^{\prime}\right) U_{2}^{\prime} \cdot\left(y U_{1}^{\prime}\right) V_{2}^{\prime}=\left(y U_{1}^{\prime} \cdot x V_{1}^{\prime}\right) W_{2}^{\prime}=(x y) W_{1} W_{2}$.

That is,

$$
\begin{aligned}
& T_{1}^{\prime} \odot T_{2}^{\prime}=\left\langle U_{1}^{\prime}, V_{1}^{\prime}, W_{1}^{\prime}\right\rangle \odot\left\langle U_{2}^{\prime}, V_{2}^{\prime}, W_{2}^{\prime}\right\rangle \\
& =\left(V_{1}^{\prime} U_{2}^{\prime}, U_{1}^{\prime} V_{2}^{\prime}, W_{1}^{\prime} W_{2}^{\prime}\right) \in A U T(Q, \cdot) .
\end{aligned}
$$

2. $T^{\prime}=<U^{\prime}, V^{\prime}, W^{\prime}>\in A A U T(Q, \cdot) \Leftrightarrow x U^{\prime} \cdot y V^{\prime}=(y \cdot x) W^{\prime}$

$$
\begin{gathered}
\Leftrightarrow(x \cdot y) W^{\prime-1}=y V^{\prime-1} \cdot x U^{\prime-1} \\
\Leftrightarrow\left\langle V^{\prime-1}, U^{\prime-1}, W^{\prime-1}\right\rangle \in A A U T(Q, \cdot) .
\end{gathered}
$$

3.

$$
\begin{gathered}
x\left(V U^{\prime}\right) \cdot y\left(U V^{\prime}\right)=(x V) U^{\prime} \cdot(y U) V^{\prime} \\
=(y U \cdot x V) W^{\prime} \\
=(y \cdot x) W W^{\prime}
\end{gathered}
$$

That is,

$$
\begin{gathered}
T \odot T^{\prime}=(U, V, W) \odot\left\langle U^{\prime}, V^{\prime}, W^{\prime}\right\rangle \\
=\left\langle V U^{\prime}, U V^{\prime}, W W^{\prime}\right\rangle \in A A U T(Q, \cdot)
\end{gathered}
$$

4. Also,

$$
\begin{gathered}
x\left(U^{\prime} U\right) \cdot y\left(V^{\prime} V\right)=\left(x U^{\prime}\right) U \cdot\left(y V^{\prime}\right) V \\
=\left(x U^{\prime} \cdot y V^{\prime}\right) W \\
=(y \cdot x) W^{\prime} W .
\end{gathered}
$$

That is, $T^{\prime} \circ T=\left\langle U^{\prime}, V^{\prime}, W^{\prime}\right\rangle \cdot(U, V, W)$

$$
=\left\langle U^{\prime} U, V^{\prime} V, W^{\prime} W\right\rangle \in A A U T(Q, \cdot) .
$$


5. We shall now verify that any two components of an anti-autotopism uniquely determines the third. Let $T_{1}=<U_{1}, V_{1}, W_{1}>, T_{2}=<$ $U_{2}, V_{2}, W_{2}>\in \operatorname{AAUT}(Q, \cdot)$, then

$$
x U_{1} \cdot y V_{1}=(y \cdot x) W_{1} \text { and } x U_{2} \cdot y V_{2}=(y \cdot x) W_{2} .
$$

Assume $U_{1}=U_{2}$ and $V_{1}=V_{2}$, then $(y \cdot x) W_{1}=(y \cdot x) W_{2} \Rightarrow W_{1}=W_{2}$. Assume $U_{1}=U_{2}$ and $W_{1}=W_{2}$, then $x U_{1} \cdot y V_{1}=x U_{2} \cdot y V_{2} \Rightarrow y V_{1}=$ $y V_{2} \Rightarrow V_{1}=V_{2}$. Assume $V_{1}=V_{2}$ and $W_{1}=W_{2}$, then $x U_{1} \cdot y V_{1}=$ $x U_{2} \cdot y V_{2} \Rightarrow x U_{1}=x U_{2} \Rightarrow U_{1}=U_{2}$.

Lemma 2.2. Let $(Q, \cdot, \backslash, /)$ be a loop.

1. The following are equivalent.

1. $(Q, \cdot, \backslash, /)$ is a middle Bol loop.

2. $\left\langle\mathbf{L}_{x}, \mathcal{R}_{x}, \mathcal{R}_{x} L_{x}\right\rangle \in \operatorname{AAUT}(Q, \cdot)$ for all $x \in Q$.

3. $\left\langle\mathbf{L}_{x}, \mathcal{R}_{x}, \mathbf{L}_{x} R_{x}\right\rangle \in \operatorname{AAUT}(Q, \cdot)$ for all $x \in Q$.

2. The following are equivalent.

1. $(Q, \cdot)$ is a left Bol loop.

2. $\left(R_{x} L_{x}, L_{x}^{-1}, L_{x}\right) \in \operatorname{AUT}(Q, \cdot) \quad$ for all $\quad x \in Q$.

3. The following are equivalent.

1. $(Q, \cdot)$ is a right Bol loop.

2. $\left(R_{x}^{-1}, L_{x} R_{x}, R_{x}\right) \in \operatorname{AUT}(Q, \cdot) \quad$ for all $\quad x \in Q$.

\section{Proof.}

1. Write the identities in Lemma 1.1 in anti-autotopic forms.

2. Write (1.2) in autotopic form.

3. Write (1.1) in autotopic form.

Theorem 2.3. A RBL $(Q, \cdot)$ is a Middle Bol loop if and only if any of the following is true: 
1. $(Q, \cdot)$ is a $L I P L$.

2. $(Q, \cdot)$ is a $L A P L$.

3. $(Q, \cdot)$ is a flexible loop.

4. $(Q, \cdot)$ is a $L B L$.

5. $(Q, \cdot)$ is a dissociative loop.

6. $(Q, \cdot)$ is a AAIPL.

7. $(Q, \cdot)$ is a Moufang loop.

8. $(Q, \cdot, \backslash, /)$ obeys the identity $x(x y \cdot x)^{-1} \cdot\left(z x^{-1}\right) \backslash x=x(z y \cdot x)^{-1} \cdot x$.

Proof. We shall need Lemma 2.1, Lemma 2.2 and Theorem 1.2.

$$
\begin{gathered}
\left(R_{x}^{-1}, L_{x} R_{x}, R_{x}\right) \odot\left\langle\mathbf{L}_{x}, \mathcal{R}_{x}, \mathbf{L}_{x} R_{x}\right\rangle=\left\langle L_{x} R_{x} \mathbf{L}_{x}, R_{x}^{-1} \mathcal{R}_{x}, R_{x} \mathbf{L}_{x} R_{x}\right\rangle \\
=\left\langle L_{x} R_{x} \mathbf{L}_{x}, \mathbf{R}_{x} \mathcal{R}_{x}, R_{x} \mathbf{L}_{x} R_{x}\right\rangle \in \operatorname{AAUT}(Q, \cdot) \Longleftrightarrow \\
x /(x y \cdot x) \cdot(z / x) \backslash x=x /(z y \cdot x) \cdot x
\end{gathered}
$$

A loop $(Q, \cdot)$ is a RIPL if and only if $a / b=a b^{-1}$ for all $a, b \in Q$. So, $(2.8)$ is true if and only if

$$
x(x y \cdot x)^{-1} \cdot\left(z x^{-1}\right) \backslash x=x(z y \cdot x)^{-1} \cdot x .
$$

Since a RBL which is also a MBL (has AAIP) is a Moufang loop, then (2.8) is equivalent to any of the properties 1 . to 7 .

Theorem 2.4. A $L B L(Q, \cdot)$ is a Middle Bol loop if and only if any of the following is true:

1. $(Q, \cdot)$ is a RIPL.

2. $(Q, \cdot)$ is a $R A P L$.

3. $(Q, \cdot)$ is a flexible loop.

4. $(Q, \cdot)$ is a $R B L$.

5. $(Q, \cdot)$ is a dissociative loop.

6. $(Q, \cdot)$ is a AAIPL.

7. $(Q, \cdot)$ is a Moufang loop.

8. $(Q, \cdot, \backslash, /)$ obeys the identity $x /\left(x^{-1} y\right) \cdot(x \cdot z x)^{-1} x=x /(x \cdot z y)^{-1} \cdot x$. 
Proof. This is similar to the proof of Theorem 2.3.

Theorem 2.5. Let $(Q, \cdot)$ be a $R B L(M B L, L B L, M B L) .(Q, \cdot)$ is a $M B L$ $(R B L, M B L, L B L)$ if and only if $(Q, \cdot)$ is a Moufang loop.

Proof. We shall need Lemma 2.1 and Lemma 2.2.

$$
\begin{gathered}
\left\langle\mathbf{L}_{x}, \mathcal{R}_{x}, \mathbf{L}_{x} R_{x}\right\rangle \circ\left(R_{x}^{-1}, L_{x} R_{x}, R_{x}\right)=\left\langle\mathbf{L}_{x} R_{x}^{-1}, \mathcal{R}_{x} L_{x} R_{x}, \mathbf{L}_{x} R_{x} R_{x}\right\rangle \\
=\left\langle\mathbf{L}_{x} \mathbf{R}_{x}, \mathcal{R}_{x} L_{x} R_{x}, \mathbf{L}_{x} R_{x}^{2}\right\rangle \in \operatorname{AAUT}(Q, \cdot) \Longleftrightarrow \\
(x / y) / x \cdot[x(z \backslash x) \cdot x]=[x /(z y)] x \cdot x \\
\Longleftrightarrow\left(x y \cdot x^{-1}\right)[x(z x) \cdot x]=[x(y z)] x \cdot x \Longleftrightarrow x y \cdot z x=x(y z) \cdot x \Longleftrightarrow
\end{gathered}
$$

$(Q, \cdot)$ is a Moufang loop. This settles the fact that if $(Q, \cdot)$ is a $\mathrm{RBL}(\mathrm{MBL})$, then $(Q, \cdot)$ is a MBL $(\mathrm{RBL})$ if and only if $(Q, \cdot)$ is a Moufang loop.

$$
\begin{gathered}
\left\langle\mathbf{L}_{x}, \mathcal{R}_{x}, \mathbf{L}_{x} R_{x}\right\rangle \circ\left(R_{x} L_{x}, L_{x}^{-1}, L_{x}\right)=\left\langle\mathbf{L}_{x} R_{x} L_{x}, \mathcal{R}_{x} L_{x}^{-1}, \mathbf{L}_{x} R_{x} L_{x}\right\rangle \\
=\left\langle\mathbf{L}_{x} R_{x} L_{x}, \mathcal{R}_{x} \mathcal{L}_{x}, \mathbf{L}_{x} R_{x} L_{x}\right\rangle \in \operatorname{AAUT}(Q, \cdot) \Longleftrightarrow \\
{[x \cdot(x / y) x][x \backslash(z \backslash x)]=[x /(z y)] x} \\
\Longleftrightarrow\left[x \cdot\left(x y^{-1}\right) x\right]\left[x^{-1}\left(z^{-1} x\right) \cdot x\right]=x \cdot\left[x\left(y^{-1} z^{-1}\right)\right] x \Longleftrightarrow x y \cdot z x=[x(y z)] x \Longleftrightarrow
\end{gathered}
$$

$(Q, \cdot)$ is a Moufang loop. This settles the fact that if $(Q, \cdot)$ is a LBL (MBL), then $(Q, \cdot)$ is a MBL $(\mathrm{LBL})$ if and only if $(Q, \cdot)$ is a Moufang loop.

\subsection{Construction of a Middle Bol Loop from a Right Bol Loop}

The Construction of a middle Bol loop from a right Bol loop is given below: Let $B=\{1,2,3, \cdots 8\}$ and let $(B, \cdot)$ be the loop with the following multiplication Table 2.1.

Then from the right Bol loop $(B, \cdot)$ with multiplication Table 2.1, a middle Bol loop $(B, \circ)$ can be obtained with the relation (1.5). The multiplication Table 2.2 represents the middle Bol loop $(B, \circ)$. 


\begin{tabular}{|c||c|c|c|c|c|c|c|c|}
\hline$\cdot$ & 1 & 2 & 3 & 4 & 5 & 6 & 7 & 8 \\
\hline \hline 1 & 1 & 2 & 3 & 4 & 5 & 6 & 7 & 8 \\
\hline 2 & 2 & 1 & 4 & 3 & 6 & 5 & 8 & 7 \\
\hline 3 & 3 & 4 & 1 & 2 & 8 & 7 & 5 & 6 \\
\hline 4 & 4 & 3 & 2 & 1 & 7 & 8 & 6 & 5 \\
\hline 5 & 5 & 6 & 7 & 8 & 1 & 2 & 3 & 4 \\
\hline 6 & 6 & 5 & 8 & 7 & 2 & 1 & 4 & 3 \\
\hline 7 & 7 & 8 & 5 & 6 & 4 & 3 & 1 & 2 \\
\hline 8 & 8 & 7 & 6 & 5 & 3 & 4 & 2 & 1 \\
\hline
\end{tabular}

Table 2.1: A right Bol Loop of order 8

\begin{tabular}{|l||l|l|l|l|l|l|l|l|}
\hline$\circ$ & 1 & 2 & 3 & 4 & 5 & 6 & 7 & 8 \\
\hline \hline 1 & 1 & 2 & 3 & 4 & 5 & 6 & 7 & 8 \\
\hline 2 & 2 & 1 & 4 & 3 & 6 & 5 & 8 & 7 \\
\hline 3 & 3 & 4 & 1 & 2 & 7 & 8 & 6 & 5 \\
\hline 4 & 4 & 3 & 2 & 1 & 8 & 7 & 5 & 6 \\
\hline 5 & 5 & 6 & 7 & 8 & 1 & 2 & 3 & 4 \\
\hline 6 & 6 & 5 & 8 & 7 & 2 & 1 & 4 & 3 \\
\hline 7 & 7 & 8 & 6 & 5 & 3 & 4 & 1 & 2 \\
\hline 8 & 8 & 7 & 5 & 6 & 4 & 3 & 2 & 1 \\
\hline
\end{tabular}

Table 2.2: A Middle Bol Loop of order 8 
Theorem 2.6. Let $(L, \cdot)$ be a right Bol loop with an $A(L)$-Holomorph $(H, \circ)$ such that $x^{-1} \cdot x \alpha \in N_{\rho}(L, \cdot) \quad$ for all $\quad \alpha \in A(L)$ and $x \in L$. Let $\odot$ be a binary operation defined on $H$ such that

$$
(\alpha, x) \odot(\beta, y)=\left(\beta \alpha,\left[y \alpha \cdot\left(x y^{-1}\right)\right] y\right) \text { for all }(\alpha, x),(\beta, y) \in H \text {. }
$$

Then, $(H, \odot)$ is a middle Bol loop.

Proof. We rely on Theorem 1.4 and the relation (1.5). Note that

$$
(\beta, y)^{-1}=\left(\beta^{-1},\left(y^{-1}\right) \beta^{-1}\right) \text { and }(\alpha, x) \circ(\beta, y)=(\alpha \beta, x \beta \cdot y) .
$$

So, $\quad(\alpha, x) \odot(\beta, y)=\left\{(\beta, y) \circ\left[(\alpha, x) \circ(\beta, y)^{-1}\right]\right\} \circ(\beta, y)$

$=\left(\beta \alpha \beta^{-1}, y \alpha \beta^{-1} \cdot\left(x \beta^{-1} \cdot\left(y \beta^{-1}\right)^{-1}\right)\right) \circ(\beta, y)$

$=\left(\beta \alpha,\left(y \alpha \cdot x y^{-1}\right) y\right)$.

\subsection{Construction of a Middle Bol Loop from a Non-abelian Group}

Theorem 2.7. Let $H$ be a subgroup of a non-abelian group $G$ and let $A=H \times G$. For $\left(h_{1}, g_{1}\right),\left(h_{2}, g_{2}\right) \in A$, define;

$$
\left(h_{1}, g_{1}\right) \square\left(h_{2}, g_{2}\right)=\left(h_{2} h_{1}, h_{2} h_{1} h_{2}^{-1} g_{2} h_{2} h_{1}^{-1} h_{2}^{-1} g_{1}\right),
$$

then $(A, \square)$ is a non-associative middle Bol loop.

Proof. We need (1.5) and Theorem 1.3. Note that $\left(h_{1}, g_{1}\right)^{-1}=\left(h_{1}^{-1}, h_{1}^{-1} g_{1}^{-1} h_{1}\right)$. Thus,

$$
\begin{aligned}
\left(h_{1}, g_{1}\right)\left[\left(h_{2}, g_{2}\right)\right. & =\left[\left(h_{2}, g_{2}\right) \circ\left(\left(h_{1}, g_{1}\right) \circ\left(h_{2}, g_{2}\right)^{-1}\right)\right] \circ\left(h_{2}, g_{2}\right) \\
& =\left[\left(h_{2}, g_{2}\right) \circ\left(h_{1} h_{2}^{-1}, h_{2}^{-1} g_{1} g_{2}^{-1} h_{2}\right)\right] \circ\left(h_{2}, g_{2}\right) \\
& =\left(h_{2} h_{1} h_{2}^{-1}, h_{1} h_{2}^{-1} g_{2} h_{2} h_{1}^{-1} h_{2}^{-1} g_{1} g_{2}^{-1} h_{2}\right) \circ\left(h_{2}, g_{2}\right) \\
& =\left(h_{2} h_{1} h_{2}^{-1} h_{2}, h_{2} h_{1} h_{2}^{-1} g_{2} h_{2} h_{1}^{-1} h_{2}^{-1} g_{1} g_{2}^{-1} h_{2} h_{2}^{-1} g_{2}\right) \\
& =\left(h_{2} h_{1}, h_{2} h_{1} h_{2}^{-1} g_{2} h_{2} h_{1}^{-1} h_{2}^{-1} g_{1}\right) .
\end{aligned}
$$

To test that this loop is not associative, let $\left(h_{1}, g_{1}\right),\left(h_{2}, g_{2}\right),\left(h_{3}, g_{3}\right) \in A$, then 


$$
\begin{aligned}
L H S= & \left(h_{1}, g_{1}\right) \square\left[\left(h_{2}, g_{2}\right) \square\left(h_{3}, g_{3}\right)\right]=\left(h_{1}, g_{1}\right) \square\left(h_{3} h_{2}, h_{3} h_{2} h_{3}^{-1} g_{3} h_{3} h_{2}^{-1} h_{3}^{-1} g_{2}\right) \\
= & \left(h_{3} h_{2} h_{1}, h_{3} h_{2} h_{1}\left(h_{3} h_{2}\right)^{-1} h_{3} h_{2} h_{3}^{-1} g_{3} h_{3} h_{2}^{-1} h_{3}^{-1} g_{2} h_{3} h_{2} h_{1}^{-1}\right. \\
& \left.\left(h_{3} h_{2}\right)^{-1} h_{3} h_{2} h_{3}^{-1} g_{3} h_{3} h_{2}^{-1} h_{3}^{-1} g_{2}\right) \\
= & \left(h_{3} h_{2} h_{1}, h_{3} h_{2} h_{1} h_{2}^{-1} h_{3}^{-1} h_{3} h_{2} h_{3}^{-1} g_{3} h_{3} h_{2}^{-1} h_{3}^{-1} g_{2} h_{3} h_{2} h_{1}^{-1} h_{2}^{-1} h_{3}^{-1}\right. \\
& \left.h_{3} h_{2} h_{3}^{-1} g_{3} h_{3} h_{2}^{-1} h_{3}^{-1} g_{2}\right) \\
= & \left(h_{3} h_{2} h_{1}, h_{3} h_{2} h_{1} h_{3}^{-1} g_{3} h_{3} h_{2}^{-1} h_{3}^{-1} g_{2} h_{3} h_{2} h_{1}^{-1} h_{3}^{-1} g_{3} h_{3} h_{2}^{-1} h_{3}^{-1} g_{2}\right) . \\
= & {\left[\left(h_{1}, g_{1}\right) \square\left(h_{2}, g_{2}\right)\right] \square\left(h_{3}, g_{3}\right)=\left(h_{2} h_{1}, h_{2} h_{1} h_{2}^{-1} g_{2} h_{2} h_{1}^{-1} h_{2}^{-1} g_{1}\right) } \\
& \square\left(h_{3}, g_{3}\right) \\
= & \left(h_{3} h_{2} h_{1}, h_{3} h_{2} h_{1} h_{3}^{-1} g_{3} h_{3}\left(h_{2} h_{1}\right)^{-1} h_{3}^{-1} h_{2} h_{1} h_{2}^{-1} g_{2} h_{2} h_{1}^{-1} h_{2}^{-1} g_{1}\right) \\
= & \left(h_{3} h_{2} h_{1}, h_{3} h_{2} h_{1} h_{3}^{-1} g_{3} h_{3} h_{1}^{-1} h_{2}^{-1} h_{3}^{-1} h_{2} h_{1} h_{2}^{-1} g_{2} h_{2} h_{1}^{-1} h_{2}^{-1} g_{1}\right) .
\end{aligned}
$$

$L H S \neq R H S$ and hence, $(A, \square)$ is a non-associative middle Bol loop.

\subsection{Construction of a Middle Bol Loop from the Ring $\mathrm{Z}_{2}$}

Theorem 2.8. Let $\left(\mathbf{Z}_{2},+, \cdot\right)$ be the ring of integers modulo 2 and $Q=\mathbf{Z}_{2}^{3}$. Define on $Q$ the operation (.) as follows:

$$
(i, j, k) \cdot(p, q, r)=(i+p, j+q, k+r+j p+j p q+i j q)
$$

$(Q, \cdot)$ is a middle Bol loop.

Proof. In (2.9), put $m=i+p, n=j+q, s=k+r+j p+j p q+i j q$, so that

$$
(i, j, k) \cdot(p, q, r)=(m, n, s) .
$$

Let $x=(i, j, k), y=(p, q, r)$ and $z=(m, n, s)$ such that $x \cdot y=z \Rightarrow x=z / y$ and $y=x \backslash z$. Then,

$$
z / y=(m, n, s) /(p, q, r)=(i, j, k)
$$

where $i=m-p, j=n-q, k=s-(r+j p+j p q+i j q)$.

$$
\begin{aligned}
k & =s-(r+(n-q) p+(n-q) p q+(m-p)(n-q) q) \\
& =s-\left(r+n p-p q+n p q-p q^{2}+(m n-m q-n p+p q) q\right) \\
& =s-\left(r+n p-p q+n p q-p q^{2}+m n q-m q^{2}-n p q+p q^{2}\right) \\
& =s-r-n p+p q-m n q+m q^{2} .
\end{aligned}
$$




$$
i=m+p, j=n+q, k=s+r+n p+p q+m n q+m q^{2} .
$$

Hence,

$(i, j, k)=\left(m+p, n+q, s+r+n p+p q+m n q+m q^{2}\right)=(m, n, s) /(p, q, r)$.

Also,

$$
x \backslash z=(i, j, k) \backslash(m, n, s)=(p, q, r)
$$

where $p=m-i, q=n-j, r=s-(k+j p+j p q+i j q)$.

$$
\begin{aligned}
r & =s-[k+j(m-i)+j(m-i)(n-j)+i j(n-j)] \\
& =s-\left[k+j m-i j+j(m n-j m-i n+i j)+i j n-i j^{2}\right] \\
& =\left(k+j m-i j+j m n-j^{2} m-i j n+i j^{2}+i j n-i j^{2}\right) \\
& =s-k-j m+i j-j m n+j^{2} m .
\end{aligned}
$$

So, $p=m+i, q=n+j, r=s+k+j m+i j+j m n+j^{2} m$.

$$
(p, q, r)=\left(m+i, n+j, s+k+j m+i j+j m n+j^{2} m\right)=(i, j, k) \backslash(m, n, s) .
$$

Now, we are ready to verify the middle Bol identity

$$
x(y z \backslash x)=(x / z)(y \backslash x) .
$$

Let $L H S=x(y z \backslash x)$, then 


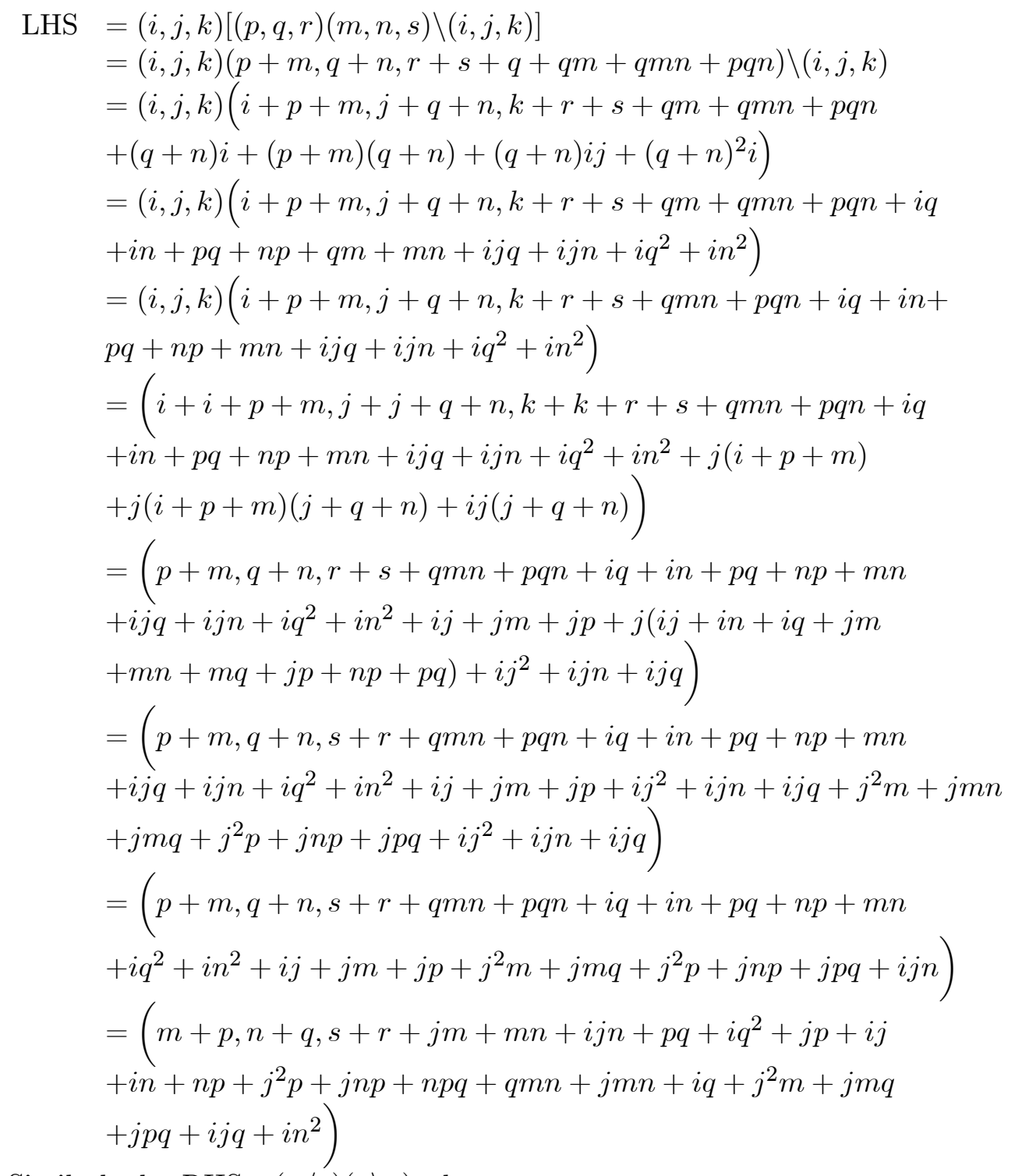

Similarly, let $\mathrm{RHS}=(x / z)(y \backslash x)$, then

$$
\begin{gathered}
\mathrm{RHS}=[(i, j, k) /(m, n, s)][(p, q, r) \backslash(i, j, k)] \\
=\left(i+m, j+n, k+s+j m+m n+i j n+i n^{2}\right)\left(i+p, j+q, k+r+q i+p q+q i j+q^{2} i\right) \\
=\left(i+m+i+p, j+n+j+q, k+s+j m+m n+i j n+p q+i q^{2}+i q+i n^{2}+k+r\right.
\end{gathered}
$$




$$
\begin{aligned}
& +i q+i j q+(j+n)(i+p)+(j+n)(i+p)(j+q)+(i+m)(j+n)(j+q)) \\
& =\left(i+m+i+p, j+n+j+q, k+s+j m+m n+i j n+p q+i q^{2}+i q+i n^{2}+i j q\right. \\
& +i j+j p+i n+n p+(i j+j p+i n+n p)(j+q)+(i j+i n+j m+m n)(j+q)) \\
& =\left(m+p, n+q, s+r+j m+m n+i j n+p q+i q^{2}+i q+i n^{2}+i j q+i j+j p+i n+n p+i j^{2}\right. \\
& \left.+j^{2} p+i j n+j n p+i j q+j p q+i q n+n p q+i j^{2}+i j n+j^{2} m+j m n+i j q+i q n+j q m+q m n\right) \\
& =\left(m+p, n+q, s+r+j m+m n+i j n+p q+i q^{2}+j p+i j+i n+n p+j^{2} p+j n p+n p q\right. \\
& \left.\quad+q m n+j m n+i q+j^{2} m+j m q+j p q+i j q+i n^{2}\right) \\
& (i, j, k)[(p, q, r)(m, n, s) \backslash(i, j, k)]=[(i, j, k) /(m, n, s)][(p, q, r) \backslash(i, j, k)] .
\end{aligned}
$$

\section{References}

[1] V. D. Belousov, Foundations of the theory of quasigroups and loops (Russian). Moskva: Nauka, 1967.

[2] R. H. Bruck, A survey of binary systems. Berlin: Springer, 1966.

[3] O. Chein, H. O. Pflugfelder, and J. D. H. Smith, Quasigroups and loops: theory and applications. Berlin: Heldermann, 1988.

[4] J. Denes and A. D. Keedwell, Latin squares and their applications. London: The English University Press, 1974.

[5] A. Drápal and V. A. Shcherbacov, "Identities and the group of isostrophisms", Commentationes Mathematicae Universitatis Carolinae, vol. 53, no. 3, pp. 347-374, 2012. [On line]. Available: https:// bit.ly/2JObrvE

[6] E. G. Goodaire, E. Jespers, and C. P. Milies, Alternative loop rings, Amsterdam: North Holland, 1996. 
[7] I. Grecu and P. Syrbu, "On some isostrophy invariants of Bol loops", Bulletin of the Transilvania University of Braşov - Series III Mathematics, Informatics, Physics, vol. 5, no. 54, pp. 145-154, 2012. [On line]. Available: https:// bit.ly/3biiO9Z

[8] I. Grecu and P. Syrbu, "Commutants of middle Bol loops", Quasigroups and related systems, vol. 22, pp. 81-88, 2014. [On line]. Available: https:/ / bit.ly/ 3ofP69g

[9] A. Gwaramija, "On a class of loops" (Russian), Uchenye Zapiski Mosgospedinstituta im. Lenina, vol. 375, pp. 25-34, 1971.

[10] T. G. Jaiyéolá, A study of new concepts in Smarandache quasigroups and loops. Ann Arbor, MI: ILQ, 2009.

[11] T. G. Jaiyéolá, S. P. David, and Y. T. Oyebo, "New algebraic properties of middle Bol loops", ROMAI journal (Online), vol. 11, no. 2, pp. 161-183, 2015. [On line]. Available: https:/ / bit.ly/ 3biTLDJ

[12] T. G. Jaiyéolá, S. P. David, E. Ilojide, and Y. T. Oyebo, "Holomorphic structure of middle Bol loops", Khayyam journal of mathematics, vol. 3, no. 2, pp. 172-184,2017, doi: 10.22034/ kjm.2017.51111

[13] E. Kuznetsov, "Gyrogroups and left gyrogroups as transversals of a special kind", Algebraic and discrete mathematics, vol. 3, pp. 54-81, 2003. [On line]. Available: https:// bit.ly/3960X1 t

[14] H. O. Pflugfelder, Quasigroups and loops: introduction. Berlin: Heldermann, 1990.

[15] D. A. Robinson, "Bol loops", Ph. D Thesis, University of WisconsinMadison, 1964.

[16] J. D. H. Smith, An introduction to quasigroups and their representations. Boca Raton, FL: Chapman \& Hall/ CRC, 2007.

[17] A. R. T. Solarin A. and B. L. Sharma, "On the construction of Bol loops", Analele ştiinţifice ale Universităţii "Al.I. Cuza" din Iaşi. Matematică, vol. 27, no. 1, pp. 13-17, 1981.

[18] P. Syrbu, "Loops with universal elasticity", Quasigroups related systems. vol. 1, pp. 57-65, 1994. [On line]. Available: https:/ / bit.ly/ 3bbb9dH 
[19] P. Syrbu, "On loops with universal elasticity", Quasigroups related systems, vol. 3, pp. 41-54, 1996. [On line]. Available: https:// bit.ly/38jT3nN

[20] P. Syrbu, "On middle Bol loops", ROMAI journal, vol. 6, no. 2, pp. 229236, 2010. [On line]. Available: https:// bit.ly/3pVGDsa

[21] P. Syrbu and I. Grecu, "On some groups related to middle Bol loops", Studia Universitatis Moldaviae: Ştiinţe Exacte şi Economice (Online), no. 7(67), pp. 10-18, 2013. [On line]. Available: https:// bit.ly/35gbpUS

[22] W. B. Vasantha Kandasamy, Smarandache loops. Rehoboth, NM: American Research Press, 2002. 